\title{
Soluble Tie2 fusion protein decreases peritoneal angiogenesis in uremic rats
}

\author{
JING XIAO $^{1 *}$, JIA GUO $^{1 *}$, XIN-XIN LIU ${ }^{1}$, XIAO-XUE ZHANG ${ }^{1}$, \\ ZHEN-ZHEN LI ${ }^{2}$, ZHAN-ZHENG ZHAO ${ }^{1}$ and ZHANG-SUO LIU ${ }^{1}$ \\ ${ }^{1}$ Department of Nephrology, and ${ }^{2}$ Key Disciplines Laboratory Clinical Medicine Henan, The First Affiliated Hospital, \\ Zhengzhou University, Zhengzhou, Henan 450052, P.R. China
}

Received December 26, 2012; Accepted April 23, 2013

DOI: $10.3892 / \mathrm{mmr} .2013 .1478$

\begin{abstract}
Angiogenesis is considered to be one of the most common mechanisms leading to ultrafiltration failure (UFF) in long-term peritoneal dialysis (PD) patients. The angiopoietin (Ang)/Tie system was found to play a role in the initiation of pathological neoangiogenesis and is also involved in peritoneal angiogenesis caused by peritoneal fluid. This aim of this study was to investigate the effects of the soluble Tie 2 fusion protein $(\mathrm{sTie} 2 / \mathrm{Fc})$ on peritoneal angiogenesis in PD-treated uremic rats. The rats were divided into 6 groups: normal, sham surgery, uremic rats without $\mathrm{PD}$, uremic PD-treated rats, uremic rats treated with $\mathrm{PD}$ and sTie $2 / \mathrm{Fc}(0.25 \mu \mathrm{g} / 100 \mathrm{~g})$ and uremic rats treated with PD and sTie $2 / F c(0.5 \mu \mathrm{g} / 100 \mathrm{~g})$. PD rats were treated once a day for 28 days prior to testing. Real-time polymerase chain reaction (RT-PCR) or tissue immunohistochemical staining was used to detect Ang-2 mRNA or protein expression in the peritoneal tissues of each group. The microvessel density (MVD) of the peritoneum was detected and quantified by immunohistochemical staining using the anti-CD34 antibody. Compared with the control group, Ang-2 mRNA and protein expression was significantly upregulated in the uremic and PD groups $(\mathrm{P}<0.05)$. MVD in the experimental group increased compared with the control group. sTie $2 / \mathrm{Fc}$ treatment decreased the levels of Ang-2 mRNA and protein expression $(\mathrm{P}<0.05)$ in a dosedependent manner and decreased PD-induced MVD in the peritoneum. In conclusion, angiogenesis of the peritoneum induced by PD was inhibited using sTie $2 / \mathrm{Fc}$ in a uremic rat model.
\end{abstract}

Correspondence to: Dr Zhan-Zheng Zhao, Department of Nephrology, The First Affiliated Hospital, Zhengzhou University, Jianshedong Road, Zhengzhou, Henan 450002, P.R. China E-mail: zzzdoctor@139.com

*Contributed equally

Key words: soluble Tie2 fusion protein, Tie2, angiopoietin-2, peritoneal dialysis, angiogenesis, ultrafiltration failure

\section{Introduction}

Peritoneal dialysis (PD) is one of the most effective ways to treat end-stage renal disease (ESRD) patients. Peritoneal structure and function are essential factors in the maintenance of PD; however, the daily instillation of PD fluid (PDF) leads to morphological and functional alterations of the peritoneal membrane. These alterations ultimately lead to ultrafiltration (UF) failure (UFF), which results in PD patients opting out of treatment, or even in mortality. For long-term PD patients, increasing angiogenesis is one of the significant changes to the peritoneum (1). According to a previous report, peritoneal angiogenesis is important in the mechanism of UFF (2).

Angiopoietin (Ang) is important in the initiation of pathological neoangiogenesis $(3,4)$. Within the Ang family, Ang-2 has been shown to be associated with neoangiogenesis (5). Under hypoxic conditions or in the presence of vascular endothelial growth factor (VEGF), Ang-2 leads to angiogenesis through promotion of the proliferation and migration of vascular endothelial cells, and by increasing the sensitivity of endothelial cells to VEGF (6,7). In the absence of VEGF, Ang-2 may lead to vessel regression (8). Ang-2 binds to the tyrosine kinase receptor Tie2, forming the Ang-2-Tie2 complex, which is involved in angiogenesis. However, there are limited reports with regard to the impact of the Ang/Tie system in peritoneal angiogenesis $(9,10)$. The extracellular Tie2 domain is released by proteases, forming soluble Tie2 (sTie2) that competitively inhibits the binding of Ang-2 to Tie2 (11).

To identify new therapeutic modalities against PD-induced angiogenesis, a uremic PD rat model treated with sTie2 fusion protein $(\mathrm{sTie} 2 / \mathrm{Fc})$ was used in this study.

\section{Materials and methods}

Reagents. Silicon-based (GB 18671-2002; Shandong Weigao Medical Polymer Co., Shandong, China) ethylene oxide sterilized PD catheters were manufactured in our laboratory $(12,13)$. Reagents used in this study were as follows: Ang-2 or CD34 polyclonal antibodies, DAB, the ultrasensitive SP kit (Maixin Inc., Fuzhou, China), total RNA extraction reagent TRIzol (Bio Basic Inc., Amherst, NY, USA), reverse transcription kit, RT-PCR amplification kit (Takara Co., Shiga, Japan), recombinant rat sTie2/Fc (R\&D 
Table I. Renal function and body weight of rats (mean $\pm \mathrm{SD}$ ).

\begin{tabular}{|c|c|c|c|c|c|}
\hline \multirow[b]{2}{*}{ Groups } & \multirow[b]{2}{*}{$\mathrm{n}$} & \multicolumn{2}{|c|}{ Serum creatinine $(\mu \mathrm{mol} / \mathrm{l})$} & \multicolumn{2}{|c|}{ Weight (g) } \\
\hline & & Day 1 & Day 28 & Day 1 & Day 28 \\
\hline Control & 8 & $24.61 \pm 4.70$ & $24.90 \pm 4.81$ & $357.80 \pm 8.49$ & $445.99 \pm 9.01$ \\
\hline Sham surgery & 8 & $24.67 \pm 4.56^{\mathrm{a}}$ & $24.36 \pm 4.49^{\mathrm{a}}$ & $357.16 \pm 9.05$ & $446.22 \pm 8.88^{a}$ \\
\hline Uremic & 8 & $66.19 \pm 6.47^{\mathrm{b}}$ & $68.94 \pm 7.70^{\mathrm{b}}$ & $358.62 \pm 8.85$ & $358.41 \pm 8.32^{b}$ \\
\hline Dialyze & 24 & $66.19 \pm 6.47^{b}$ & $62.30 \pm 5.88^{\mathrm{b}}$ & $409.90 \pm 10.21$ & $436.93 \pm 8.11^{\mathrm{c}}$ \\
\hline
\end{tabular}

${ }^{\mathrm{a}} \mathrm{P}>0.05$ and ${ }^{\mathrm{b}} \mathrm{P}<0.05$ compared with the control group; ${ }^{\mathrm{c}} \mathrm{P}<0.05$ compared with the uremic groups.

systems, Minneapolis, MN, USA), and $4.25 \%$ peritoneal dialysate (Baxter, Deerfield, IL, USA).

Model establishment and group distribution. This study was approved by Experimental Animal Center of Medical School, Zhengzhou University (Zhengzhou, Henan). We purchased 48 male Sprague-Dawley rats from the Experimental Animal Center of the Medical School, Zhengzhou University (Zhengzhou, China), with initial weights of 180-200 g. The rats had free access to food and water throughout the study. The rats were randomly divided into 6 groups: normal rats as a control group (control, $\mathrm{n}=8$ ); rats with sham surgery (sham, $\mathrm{n}=8$ ); uremic rats without PD (uremic, $\mathrm{n}=8$ ); uremic rats dialyzed with $4.25 \%$ PD solution (dialyze, $n=8$ ); uremic rats dialyzed with $4.25 \%$ PD solution and treated with subcutaneous injections of $0.25 \mu \mathrm{g} / 100 \mathrm{~g}$ sTie $2 / \mathrm{Fc}$ (dialyze $+0.25 \mathrm{Tie} 2$, $\mathrm{n}=8$ ); and uremic rats dialyzed with $4.25 \%$ PD solution and treated by subcutaneous injections of $0.50 \mu \mathrm{g} / 100 \mathrm{~g}$ sTie $2 / \mathrm{Fc}$ (dialyze $+0.50 \mathrm{Tie} 2, \mathrm{n}=8)$. The rats in the sham surgery group underwent removal of the renal capsule from both kidneys.

Rats in the uremic groups were subjected to a 5/6 nephrectomy, performed by removing the upper and lower thirds of the left kidney, and then the removed renal tissue was weighed. One week later, the entirety of the right kidney was resected (14).

Five weeks after the subtotal nephrectomy, a peritoneal catheter, connected to a subcutaneous mini-vascular access port, was implanted into rats of the PD groups $(10,15)$. One week after implantation, the PD rats were infused with $4.25 \%$ PD solution ( $3 \mathrm{ml} / 100 \mathrm{~g}$ body weight) each day and the PD fluid was retained for $2 \mathrm{~h}$ before draining.

STie $2 / F c$ was administered every day during PD treatment, for a total of 14 doses. After 28 days of regular PD treatment, all rats were sacrificed and the peritoneal tissues were collected.

Real-time polymerase chain reaction (RT-PCR) and tissue immunohistochemical staining were used to detect Ang-2 mRNA and protein expression in peritoneal tissues in each rat. The microvessel density (MVD) of the peritoneum was detected and quantified with immunohistochemical staining using anti-CD34 antibody.

Detection of Ang-2 protein and MVD in rat peritoneal tissue. Tissue specimens were embedded in paraffin and $5-\mu \mathrm{m}$ sections were obtained. From each rat, one section of the peritoneum was randomly selected for immunohistochemical pathological examination to detect Ang-2 protein expression and MVD.

Specimens were dewaxed and rehydrated, treated with $3 \% \mathrm{H}_{2} \mathrm{O}_{2}$ to block endogenous peroxidase and incubated in $5 \%$ goat antiserum for $30 \mathrm{~min}$ at $37^{\circ} \mathrm{C}$. The sections were marked with Ang-2 antibody (1:80 dilution) and incubated overnight at $4{ }^{\circ} \mathrm{C}$. The sections were washed once with PBS $(0.01 \mathrm{M}, \mathrm{pH}$ 7.4), then a biotin-labeled secondary antibody was added and incubated at $37^{\circ} \mathrm{C}$ for $35 \mathrm{~min}$. Avidin biotin-peroxidase complex and DAB were used for the color reaction. PBS (0.01 M, pH 7.4) without primary antibody was used as a negative control (16).

Detection of Ang-2 mRNA in peritoneal tissues. Total RNA was isolated with a single-step method using TRIzol reagent, according to the manufacturer's instructions (17). An ultraviolet spectrophotometer XO-1101 (Kai-Di high-speed analytical instruments Co., Ltd., Nanjing, China) was used to measure the total RNA concentration. Complementary DNA (cDNA) was synthesized according to standard procedures (18). The primers were as follows: Ang-2 (100 bp product) sense, 5'-CGGCCACAGTCAACAACTCA-3' and antisense, 5'-GCTCTTATAGTCGGGCGATGA-3'; and $\beta$-actin (222 bp product) sense, 5'-AGCCATGTACGTAGCCATCC-3' and antisense, 5'-GCTGTGGGCGTGAAGCTGTA-3'.

Electrophoresis was performed and imaging system analysis software was used to capture images and determine the absorbance (A) value (Bio-Rad, Berkeley, CA, USA). Ang-2 mRNA was expressed as the ratio of its amplicon to the $\beta$-actin amplicon and analyzed by densitometry using Image J software (National Institutes of Health, Bethesda, MD, USA).

Immunohistochemical staining for CD34 and MVD value. The omentum was removed from the sacrificed rats and fixed in a sufficient amount of $4 \%$ phosphate-buffered formaldehyde for $24 \mathrm{~h}$. Tissue samples were embedded in paraffin and $4-\mu \mathrm{m}$ sections were cut. Cut sections were stained for immunohistochemical examination with CD34-related antigen antibody (1:100 dilution).

The MVD was assessed according to Weidner (19), and the microscopic image was fixed at the position with the greatest amount of vessels at x100 magnification. Vessels were counted in five random fields at $\times 200$ magnification and the average number of microvessels was assessed. A single CD34-positive 


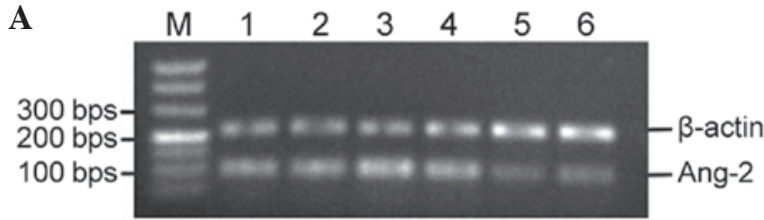

B

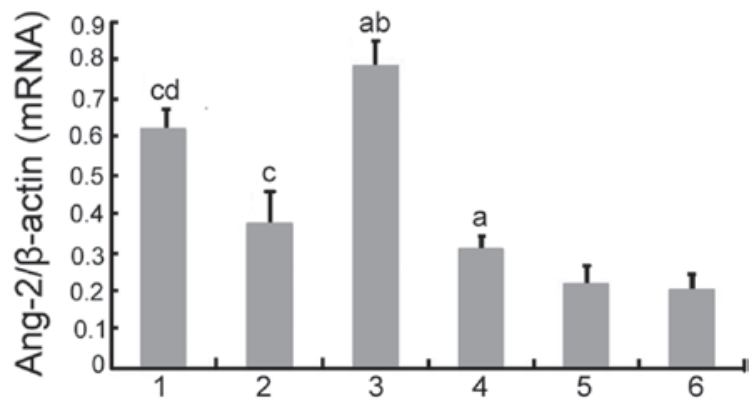

Figure 1. Ang-2 mRNA was assessed in the peritoneal tissue by RT-PCR. (A) Representative RT-PCR of Ang-2 mRNA (100 bps) and $\beta$-actin (222 bps). Lanes: (1) dialyze + 0.25Tie2 group; (2) dialyze + 0.50Tie2 group; (3) dialyze group; (4) uremic group; (5) sham surgery group; (6) control group; and (M) DNA ladder. (B) Semi-quantification demonstrating that Ang-2 mRNA increased. $\mathrm{n}=8$; ${ }^{\mathrm{a}} \mathrm{P}<0.05$ compared with controls; ${ }^{\mathrm{b}} \mathrm{P}<0.05$ compared with the dialyze group; ${ }^{\mathrm{C}} \mathrm{P}<0.05$ compared with the uremic group; ${ }^{\mathrm{d}} \mathrm{P}<0.05$ compared with the dialyze +0.50 Tie 2 group. Ang-2, angiopoietin-2; RT-PCR, real-time polymerase chain reaction; sTie $2 / F c$, soluble Tie2 fusion protein; 0.25Tie2, $0.25 \mu \mathrm{g} / 100 \mathrm{~g}$ sTie2/Fc; 0.50Tie2, $0.50 \mu \mathrm{g} / 100 \mathrm{~g} \mathrm{sTie} 2 / \mathrm{Fc}$.

$\mathbf{A}$

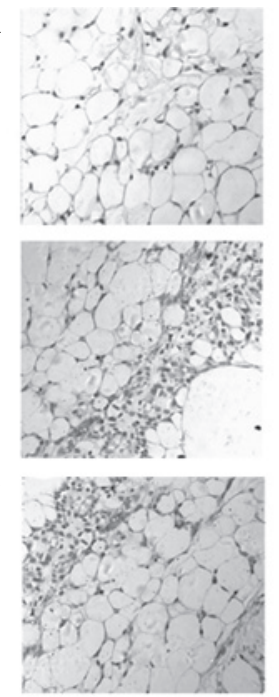

D
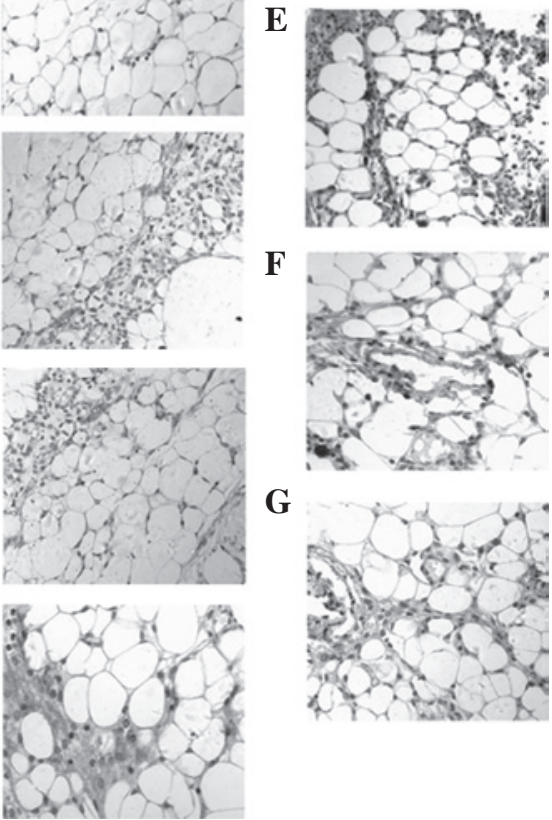

F

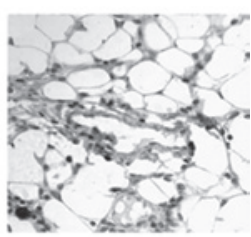

G

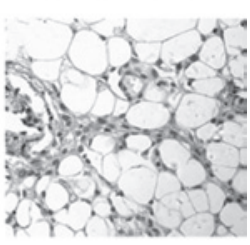

Figure 2. Ang-2 protein was assessed in the peritoneal tissue by immunohistochemistry (magnification, x200). (A) Negative control; (B) Control group; (C) Sham surgery group; (D) Uremic group; (E) Dialyze group; (F) Dialyze + 0.25Tie2 group; (G) Dialyze + 0.50Tie2 group. Ang-2, angiopoietin-2; sTie2/Fc, soluble Tie2 fusion protein; $0.25 \mathrm{Tie} 2,0.25 \mu \mathrm{g} / 100 \mathrm{~g}$ sTie2/Fc; 0.50Tie2, $0.50 \mu \mathrm{g} / 100 \mathrm{~g} \mathrm{sTie} / \mathrm{Fc}$.

cell, or a cluster of endothelial cells clearly separated from adjacent microvessels and other connective tissue elements were considered to be vessels.
A

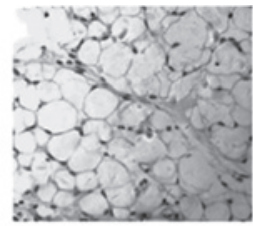

B

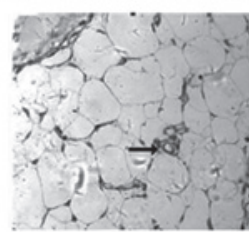

C

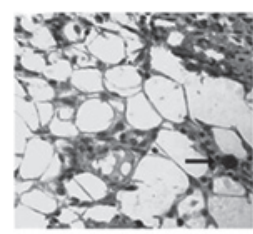

D

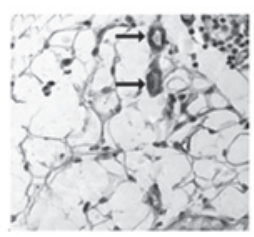

E

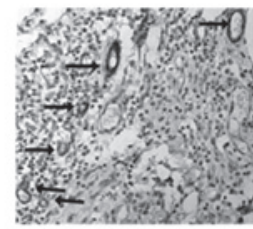

$\mathbf{F}$

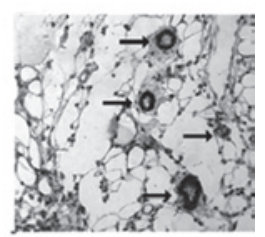

G
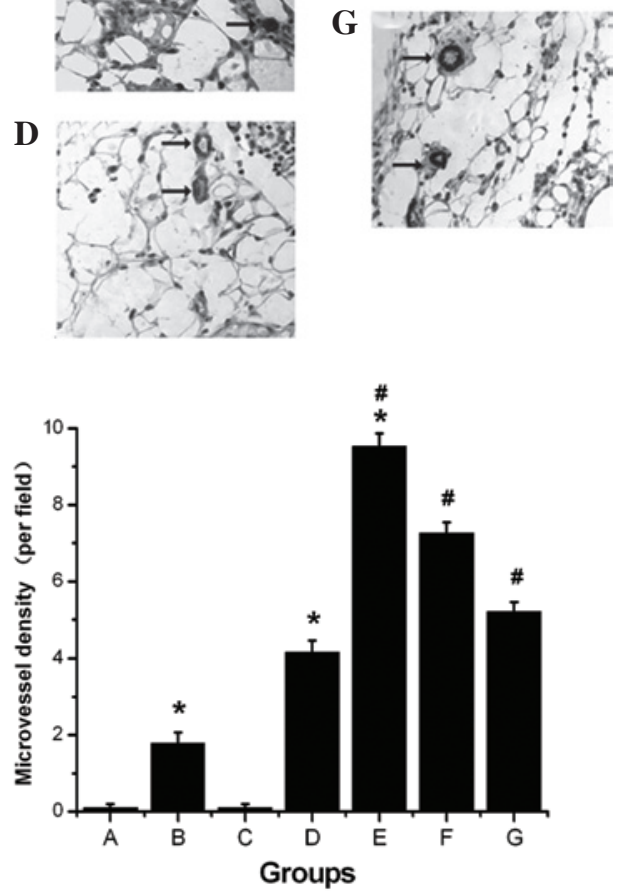

Figure 3. Microvessel density (MVD) value assessed in peritoneal tissue by anti-CD34 immunohistochemistry. Upper: (magnification, x200). (A) Negative control; (B) Control group; (C) Sham operation group; (D) Uremic group; (E) Dialyze group; (F) Dialyze+0.25Tie2 group; (G) Dialyze+0.50Tie2 group. Lower: MVD quantification. " $\mathrm{P}<0.05$ compared with the B, E and D groups. ${ }^{\text {P }} \mathrm{P}<0.05$ compared with the $\mathrm{E}, \mathrm{F}$ and $\mathrm{G}$ groups. sTie2/Fc, soluble Tie2 fusion protein; $0.25 \mathrm{Tie} 2,0.25 \mu \mathrm{g} / 100 \mathrm{~g}$ sTie2/ Fc; 0.50Tie2, $0.50 \mu \mathrm{g} / 100 \mathrm{~g} \mathrm{sTie} 2 / \mathrm{Fc}$.

Statistical analysis. Data were expressed as the mean \pm SD. Multiple comparisons were initially subjected to one-way analysis of variance (ANOVA). Correlations were analyzed using the Pearson's correlation test. Statistical analysis was performed using SPSS13.0 software (SPSS Inc., Chicago, IL, USA). P $<0.05$ was considered to indicate a statistically significant difference.

\section{Results}

Renal function of the uremic rat model. There were no statistically significant differences in the serum creatinine ( $\mathrm{SCr}$ ) levels between the control and sham surgery groups. However, serum creatinine levels were increased 2-3-fold in uremic rats $(\mathrm{P}<0.05)$ compared with the control group (Table I). 


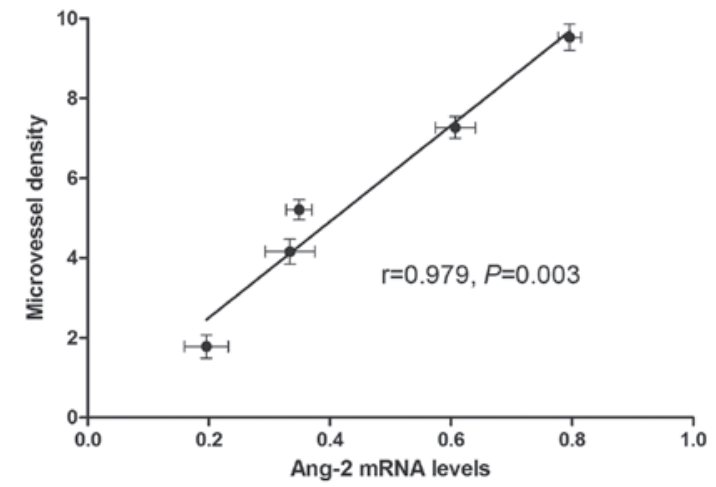

Figure 4. Correlation between Ang-2 mRNA levels and microvessel density across groups. Ang-2, angiopoietin-2.

Effects of recombinant rat sTie2/Fc on the expression of Ang-2 in peritoneal tissue. Ang-2 mRNA and protein expression was observed in each group. There were no significant differences in Ang-2 mRNA and protein expression between the control and sham surgery groups. RT-PCR demonstrated that there was a significant difference in Ang-2 mRNA expression between the control, uremic and dialyze groups (0.196 \pm 0.036 vs. $0.334 \pm 0.041$ vs. $0.796 \pm 0.019$, respectively; $\mathrm{P}<0.05)$. Furthermore, Ang-2 mRNA expression was significantly different between the dialyze, dialyze $+0.25 \mathrm{Tie} 2$ and dialyze +0.50 Tie2 groups $(0.796 \pm 0.019$ vs. $0.607 \pm 0.033$ vs. $0.349 \pm 0.021$, respectively; $\mathrm{P}<0.05$; Fig. 1). Ang-2 protein expression was observed in peritoneal capillary endothelial cells and in the cytoplasm of the peritoneal mesothelial cells using immunohistochemistry. Of all the groups, staining in the dialyze group was the strongest, whilst it was weakest in the dialyze +0.50 Tie2 group (Fig. 2).

Effects of recombinant rat sTie $2 / F c$ on MVD. Few new microvessels were observed in the control and sham surgery groups. However, there was a significant difference in the MVD between control, uremic and dialyze groups $(1.78 \pm 0.29$ vs. $4.16 \pm 0.31$ vs. $9.53 \pm 0.33$, respectively; $\mathrm{P}<0.05)$. Moreover, there was a significant difference in the MVD between the dialyze, dialyze +0.25 Tie2 and dialyze +0.50 Tie 2 groups (9.53 \pm 0.33 vs. $7.27 \pm 0.27$ vs. $5.21 \pm 0.25$, respectively; $\mathrm{P}<0.05$; Fig. 3).

Spearman results. A significant positive correlation was observed between Ang-2 mRNA expression and MVD ( $r=0.9790 ; \mathrm{P}=0.003$; Fig. 4).

\section{Discussion}

$\mathrm{UFF}$ is one of the main reasons that long-term PD patients choose to discontinue their treatment (20). Peritoneal angiogenesis is considered to be the most probable mechanism leading to UFF (1). Increases in peritoneal angiogenesis lead to an increase in peritoneal vascular surface area, which accelerates the transport of small soluble molecules; this causes rapid disappearance of the osmotic gradient, thus inducing the loss of the peritoneal UF capacity. These events may predict not only technical failure, but also patient mortality $(21,22)$. Studies have revealed that anti-angiogenic treatments, such as angiostatin, slow the process of peritoneal angiogenesis, which is a more effective method of protecting the peritoneal membrane function than directly inhibiting peritoneal fibrosis (23). As with any disease, early intervention, diagnosis and treatment may improve the outcome for PD patients.

In 1996, Hanahan and Folkman (24) proposed a hypothesis on the balance of the angiogenesis switch, where angiogenesis is regulated by an equilibrium between inducers and inhibitors. Once the balance is broken, the endothelial cells are activated and promote neoangiogenesis. Other studies have demonstrated that in a uremic and high-glucose environment, the levels of certain inducers (including the fibroblast growth factor and VEGF) were raised in the peritoneum and positively correlated with angiogenesis (24-27). Inhibitors (including thrombospondin-1, $16 \mathrm{kD}$ prolactin, interferon $\alpha / \beta$, platelet factor- 4 and angiostatin) are able to inhibit peritoneal neoangiogensis by downregulating the expression of inducer factors in the peritoneum $(24,27)$.

It has been demonstrated that the Ang/Tie pathway is an inducer factor in vasculogenesis and angiogenesis, but little is known about its involvement in peritoneal angiogenesis. One study confirmed increased angiogenesis and fibrosis in the uremic rat model under PD, and suggested that this increase was due to increased Ang-2 levels associated with decreased Tie2, indicating that this pathway may be targeted in order to preserve the peritoneum (9). The present study revealed that in a uremic and high-glucose environment, Ang-2 mRNA and protein expression increased in peritoneal tissues, indicating that upregulation of Ang-2 was the likely cause of peritoneal angiogenesis. Furthermore, we observed a positive correlation between Ang-2 and peritoneal angiogenesis.

A previous study (27) reported that soluble receptors for angiogenic growth factors, which were used as endogenous ligand antagonists, may be useful in inhibiting signal transduction, thus influencing the biological action of ligands. Studies showed that the Ang/Tie2 system was involved in cancer tissue and retinal neovascularization, and that the use of a sTie-2/Fc effectively inhibited angiogenesis $(28,29)$. Results of the present study are consistent with a previous study (9) demonstrating that the Ang/Tie2 system is upregulated in a uremic rat model treated with PD. Results suggest that inhibiting this pathway may be an effective method of preventing peritoneal angiogenesis, allowing PD patients to continue with their treatment and improving survival rates. This study demonstrated that sTie-2/Fc may be used to effectively inhibit peritoneal angiogenesis. VEGF and VEGF receptor inhibitors have been approved for angiogenesis inhibition in a number of studies, but their use alone is unable to completely prevent angiogenesis $(28,30)$. As VEGF is also upregulated in uremic patients under PD (31), the combined use of a VEGF inhibitor and sTie-2/Fc may be an effective method to reduce angiogenesis in PD patients (28).

In conclusion, results from this study suggest that targeting Ang-2 using a soluble Tie-2 receptor may be an effective method for preventing peritoneal angiogenesis in uremic patients undergoing PD. This study also provides an experimental basis for the development of a future clinical treatment using sTie-2/Fc. 


\section{References}

1. van Westrhenen R, Zweers MM, Kunne C, de Waart DR, van der Wal AC and Krediet RT: A pyruvate-buffered dialysis fluid induces less peritoneal angiogenesis and fibrosis than a conventional solution. Perit Dial Int 28: 487-496, 2008.

2. Saxena R: Pathogenesis and treatment of peritoneal membrane failure. Pediatr Nephrol 23: 695-703, 2008.

3. Ramsauer M and D'Amore PA: Getting Tie(2)d up in angiogenesis. J Clin Invest 110: 1615-1617, 2002.

4. Koh GY, Kim I, Kwak HJ, Yun MJ and Leem JC: Biomedical significance of endothelial cell specific growth factor, angiopoietin. Exp Mol Med 34: 1-11, 2002.

5. Guo P, Imanishi Y, Cackowski FC, et al: Up-regulation of angiopoietin-2, matrix metalloprotease-2, membrane type 1 metalloprotease, and laminin 5 gamma 2 correlates with the invasiveness of human glioma. Am J Pathol 166: 877-890, 2005

6. Reiss Y: Angiopoietins. Recent Results Cancer Res 180:3-13,2010

7. Shin HY, Kim JH, Phi JH, et al: Endogenous neurogenesis and neovascularization in the neocortex of the rat after focal cerebral ischemia. J Neurosci Res 86: 356-367, 2008.

8. Mandriota SJ and Pepper MS: Regulation of angiopoietin-2 mRNA levels in bovine microvascular endothelial cells by cytokines and hypoxia. Circ Res 83: 852-859, 1998.

9. Yuan J, Fang W, Lin A, Ni Z and Qian J: Angiopoietin-2/Tie2 signaling involved in TNF- $\alpha$ induced peritoneal angiogenesis Int J Artif Organs 35: 655-662, 2012.

10. Yuan J, Fang W, Ni Z, et al: Peritoneal morphologic changes in a peritoneal dialysis rat model correlate with angiopoietin/Tie-2. Pediatr Nephrol 24: 163-170, 2009.

11. Lin P, Polverini P, Dewhirst M, Shan S, Rao PS and Peters $\mathrm{K}$ : Inhibition of tumor angiogenesis using a soluble receptor establishes a role for Tie2 in pathologic vascular growth. J Clin Invest 100: 2072-2078, 1997.

12. Zhao ZZ, Cao Y, Liu ZS, et al: Effects of recombinant human endostatin on peritoneal angiogenesis in peritoneal dialysis rats. Nephrology (Carlton) 16: 599-606, 2011.

13. Gao D, Zhao ZZ, Liang XH, Li Y, Cao Y and Liu ZS: Effect of peritoneal dialysis on expression of vascular endothelial growth factor, basic fibroblast growth factor and endostatin of the peritoneum in peritoneal dialysis patients. Nephrology (Carlton) 16: 736-742, 2011.

14. Ghosh SS, Krieg RJ, Sica DA, Wang R, Fakhry I and Gehr T: Cardiac hypertrophy in neonatal nephrectomized rats: the role of the sympathetic nervous system. Pediatr Nephrol 24: 367-377, 2009.

15. Zhang XD and Qian JQ: An improved animal model of peritoneal dialysis mimicked human peritoneal dialysis. Chin J Blood Purif 4: 326-328, 2005.

16. Bunone G, Vigneri P, Mariani L, et al: Expression of angiogenesis stimulators and inhibitors in human thyroid tumors and correlation with clinical pathological features. Am J Pathol 155: 1967-1976, 1999.
17. Deng MY, Wang H, Ward GB, Beckham TR and McKenna TS: Comparison of six RNA extraction methods for the detection of classical swine fever virus by real-time and conventional reverse transcription-PCR. J Vet Diagn Invest 17: 574-578, 2005.

18. Sambrook J and Russell DW: Molecular Cloning: A Laboratory Manual. 3rd edition. CSHL Press, Cold Spring Harbor, NY, 2001.

19. Weidner N: Intratumor microvessel density as a prognostic factor in cancer. Am J Pathol 147: 9-19, 1995

20. Chaudhary K: Peritoneal dialysis drop-out: causes and prevention strategies. Int J Nephrol 2011: 434608, 2011.

21. Noh H, Kim JS, Han KH, et al: Oxidative stress during peritoneal dialysis: implications in functional and structural changes in the membrane. Kidney Int 69: 2022-2028, 2006.

22. Parikova A, Smit W, Struijk DG and Krediet RT: Analysis of fluid transport pathways and their determinants in peritoneal dialysis patients with ultrafiltration failure. Kidney Int 70: 1988-1994, 2006.

23. Margetts PJ, Gyorffy S, Kolb M, et al: Antiangiogenic and antifibrotic gene therapy in a chronic infusion model of peritoneal dialysis in rats. J Am Soc Nephrol 13: 721-728, 2002.

24. Hanahan D and Folkman J: Patterns and emerging mechanisms of the angiogenic switch during tumorigenesis. Cell 86: 353-364, 1996.

25. Zhao ZZ, Liu ZS and Liang XH: Expression and significance of aquaporin and basic fibroblast growth factor in human peritoneal mesothelial cells. Chin J Nephrol 24: 669-670, 2008.

26. Zhao ZZ, Cao Y and Liu ZS: The effects of recombinant human edostatin on peritoneul angiogenesis in peritoneal dialysis rats. Chin J Nephrol 29: 791-795, 2010.

27. Donnelly R and Yeung JM: Therapeutic angiogenesis: a step forward in intermittent claudication. Lancet 359: 2048-2050, 2002.

28. Takagi H, Koyama S, Seike H, et al: Potential role of the angiopoietin/tie2 system in ischemia-induced retinal neovascularization. Invest Ophthalmol Vis Sci 44: 393-402, 2003

29. Zhang H and Liu ZL: Suppression of experimental choroidal neovascularization by soluble Tie2 fusion protein. Int J Ophthalmol 8: 272-274, 2008.

30. Shibuya M: Vascular endothelial growth factor-dependent and -independent regulation of angiogenesis. BMB Rep 41: 278-286, 2008.

31. Selgas R, del Peso G, Bajo MA, et al: Vascular endothelial growth factor (VEGF) levels in peritoneal dialysis effluent. J Nephrol 14: 270-274, 2001. 